\title{
Análisis de los métodos de enseñanza que usa profesorado destacado en clases de ciencias sociales ${ }^{1}$
}

\author{
Analysis of Teaching Methods That Outstanding Professors Use in Social Science ${ }^{2}$
}

\section{Análise de métodos de ensino utilizados por docentes proeminentes nas aulas de ciências sociais ${ }^{3}$}

Marta Castañeda-Meneses

Universidad de Playa Ancha

Valparaíso, Chile

marta.castaneda@upla.cl

https://orcid.org/0000-0001-9617-4408

\begin{abstract}
Resumen: El objetivo del estudio es analizar los métodos de enseñanza utilizados por profesorado del nivel de educación primaria reconocido como destacado por el Ministerio de Educación chileno desde la perspectiva que brindan los conceptos sociales claves. El estudio, desde una visión cualitativa, utiliza como técnica de recogida inicial de la información un focus group y, principalmente, se centra en la observación no participante de clases de historia, geografía y ciencias sociales. El colectivo está formado por personal docente del sistema escolar reconocido como destacado. Los datos son analizados desde la perspectiva de los conceptos sociales claves, en este caso: tiempo, espacio, convivencia, identidad y cambio, y desde los métodos de enseñanza utilizados. Como principal conclusión del estudio se puede mencionar que los modelos de enseñanza evidencian una perspectiva tradicional, asociada principalmente a un método expositivo, opción metodológica que no descarta al estudiantado, quien, desde un rol participativo, cede el protagonismo al personal docente en su rol de guía del aprendizaje.
\end{abstract}

Palabras claves: Docente de escuela primaria; enseñanza de las ciencias sociales; método de enseñanza.

\footnotetext{
${ }^{1}$ Este artículo surge del Proyecto de Investigación EDU 10 16-17 financiado por la Universidad de Playa Ancha.

${ }^{2}$ This article is derived from the Research Project EDU 10 16-17 funded by the University of Playa Ancha.

${ }^{3}$ Este artigo é derivado do Projeto EDU 10 16-17, financiado pela Universidade de Playa Ancha.
} 
doi: http://dx.doi.org/10.15359/ree.23-3.18

URL: http://www.una.ac.cr/educare

CORREO: educare@una.cr

\begin{abstract}
The study aims to analyze teaching methods used by primary school teachers recognized as outstanding by the Chilean Ministry of Education from the perspective provided by key social concepts. From a qualitative point of view, the study uses a focus group as an initial information gathering technique and, mainly, focuses on non-participating observation of history, geography, and social science classes. The group is made up of teachers from the school system who are recognized as outstanding. The data are analyzed from the perspective of key social concepts, in this case: time, space, coexistence, identity and change, and from the teaching methods used. The main conclusion of the study is that the teaching models show a traditional perspective, mainly associated with an expository method, a methodological option that does not rule out the student body, which, from a participatory role, cedes the protagonism to the teaching staff in their role as a guide to learning.
\end{abstract}

Keywords: Primary school teachers; social science teaching; teaching methods.

Resumo: O objetivo do estudo é analisar os métodos de ensino utilizados por docentes no nível do ensino fundamental, que foram reconhecidos pelo Ministério da Educação do Chile, a partir da perspectiva proporcionada sobre os principais conceitos sociais. O estudo, de uma perspectiva qualitativa, utiliza como técnica de coleta inicial de informações um grupo focal e, principalmente, enfoca a observação não participante das aulas de história, geografia e ciências sociais. O grupo é composto por docentes do sistema escolar reconhecidos como excepcionais. Os dados são analisados a partir da perspectiva de conceitos sociais fundamentais, neste caso: tempo, espaço, coexistência, identidade e mudança, a partir dos métodos de ensino utilizados. Como principal conclusão do estudo pode-se mencionar que os modelos de ensino apresentam uma perspectiva tradicional, associada principalmente a um método expositivo, opção metodológica que não descarta o grupo de estudantes, que, a partir de um papel participativo, atribui o protagonismo ao corpo docente em sua função de guiar a aprendizagem.

Palavras-chave: Docentes de ensino fundamental; ensino das ciências sociais; método de ensino

\title{
Introducción
}

ElMinisterio de Educación deChile (Mineduc, 2003) describe, en el documento denominado Marco de la Buena Enseñanza, las tareas profesionales propias de la función docente. En este instrumento considerado orientador de la tarea del profesorado, según la Resolución 11.205 de 2004b (Mineduc) reconoce cuatro áreas prioritarias: preparación de la enseñanza, creación de un ambiente propicio para el aprendizaje, enseñanza para el aprendizaje de todo el estudiantado y responsabilidades profesionales. El Marco de la Buena Enseñanza es el referente utilizado en los procesos de evaluación de profesores y profesoras del ámbito municipal, es decir, de quienes se desempeñan en docencia de aula, en establecimientos que dependen económicamente del gobierno para su funcionamiento.

El proceso evaluativo del personal docente del ámbito municipal, según Mineduc (2004a), posee un "carácter formativo, orientado a mejorar la labor pedagógica de los educadores y a promover su desarrollo profesional continuo" (p. 1). En su implementación se consideran 
variadas evidencias de desempeño profesional y del dominio conceptual de las disciplinas impartidas. Los resultados del personal docente que participó del proceso los clasifican en cuatro categorías acorde a su desempeño: destacado, competente, básico e insatisfactorio.

De acuerdo con la normativa vigente, un profesor o profesora de desempeño profesional destacado es aquel que es claro, consistente y sobresale con respecto a lo que se espera para el conjunto de los indicadores evaluados. Un desempeño profesional competente reconoce a quien cumple con regularidad el conjunto de los indicadores evaluados. Un desempeño profesional con cierta irregularidad se califica como desempeño básico y, finalmente, un desempeño profesional insatisfactorio se asocia a quien presenta claras debilidades en su función profesional (Mineduc, 2004a).

Por otrolado, los Programas de Estudio de Historia, Geografía y Ciencias Sociales especifican como objetivo central para el nivel de la adquisición del estudiantado de "sentido de identidad y de pertenencia a la sociedad... comprender su cultura, apropiarse de ella y participar en su construcción" (Mineduc, 2012, p. 1). Este sentido de identidad, de comprensión de la cultura y de su proyección hacia la comprensión de otras, no solo se relaciona con reconocer la responsabilidad profesional en el área, sino que permite organizar las propuestas de enseñanza en torno a los conceptos sociales que subyacen a estas propuestas.

El desarrollo conceptual se refiere tanto al tipo de características o atributos con los que se definen los conceptos, como a las conexiones que se establecen entre ellos. El mismo progresa desde la comprensión de los conceptos a través de sus dimensiones más concretas, hasta la asignación de sus cualidades más abstractas. (Carretero, Castorina, Sarti, Van Alphen y Barreiro, 2013, p. 14)

Para Pozo (1992), aprender conceptos sociales implica establecer una relación con los conocimientos anteriores, lo que se alcanza por la comprensión de este mismo, el cual se adquiere gradualmente. Santisteban (2010) precisa que el aprendizaje de conceptos se caracteriza por su progresión geométrica, su potencialidad permite más interrelaciones conceptuales y mejores aprendizajes. Los conceptos requieren de comprensión significativa.

Los conceptos sociales, desde esta perspectiva no aluden exclusivamente a la mera definición o caracterización de un concepto, o a la forma como es aprendido, sino que se convierten en oportunidades de adquisición del conocimiento social que supera al aprendizaje memorístico; se pueden llegar a identificar algunos de ellos como conceptos sociales claves (Benejam, 1997; Carretero et al., 2013; Martorella, 1993; Taba, 1971; Tribó, 1999). A partir del análisis de los conceptos sociales claves se pueden definir en los siguientes términos: 
doi: http://dx.doi.org/10.15359/ree.23-3.18

URL: http://www.una.ac.cr/educare

CORREO: educare@una.cr

Conocimientos desarrollados por las ciencias sociales y seleccionados como contenidos para su enseñanza y aprendizaje en la realidad escolar. Permiten establecer las diferencias identitarias básicas, aunque no excluyentes, entre la enseñanza de las ciencias sociales y otras áreas del conocimiento. Involucran tanto conceptos generales como subordinados, los que representan redes temáticas y/o conceptuales de complejidad creciente, que contribuyen a la comprensión de la sociedad. (Castañeda, 2016, p. 43)

Los referentes anteriores permiten plantear el objetivo de investigación del que da cuenta este artículo: Analizar los métodos de enseñanza utilizados por el profesorado destacado del nivel de educación primaria desde la perspectiva de los conceptos sociales claves. Según los criterios propuestos por Hernández, Fernández y Baptista (2014), la investigación responde principalmente al criterio de conveniencia, puesto que se busca analizar los métodos de enseñanza con un enfoque, que no es utilizado habitualmente, conceptos sociales claves, permitiendo la generación de nuevas perspectivas de análisis.

\section{Referentes conceptuales}

La investigación se da en el contexto de la evaluación docente, la cual se encuentra regulada según el Decreto Ley N.0192, 2004 del Ministerio de Educación. Esta norma legal define la evaluación docente como el "sistema de evaluación de los profesionales de la educación que se desempeñen en funciones de docencia de aula, de carácter formativo, orientado a mejorar la labor pedagógica de los educadores y a promover su desarrollo profesional continuo" (Mineduc, 2004a, p. 1). Esta misma normativa reconoce como docentes, susceptibles de evaluación a profesionales de la educación que ejercen funciones de aula en establecimientos del sector municipal. Este proceso se lleva a cabo cada cuatro años, salvo en aquellos casos cuyos resultados son insatisfactorios.

El principal referente de evaluación docente lo constituye el Marco de la Buena Enseñanza, documento que a partir de la descripción realizada de la labor profesional es la base que permite la construcción de los instrumentos utilizados en el proceso. La evidencia que recibe mayor ponderación en la evaluación docente es el denominado portafolio de desempeño pedagógico. Acorde al citado decreto Ley N. ${ }^{\circ}$ 192, artículo 14 del año 2004, el portafolio tiene como función:

Recoger, a través de productos estandarizados por el Centro de Perfeccionamiento, Experimentación e Investigaciones Pedagógicas (CPEIP), evidencia verificable respecto a las mejores prácticas de desempeño del docente evaluado. Deberá contemplar productos escritos, en los cuales el docente debe reportar distintos aspectos de su quehacer profesional. (Mineduc 2004a, p. 5) 
Es de particular interés en esta investigación aquel profesorado que es considerado como destacado en el proceso. Según se consigna en el artículo 9 del decreto 192:

un desempeño profesional [destacado] ..., es claro, consistente y sobresale con respecto a lo que se espera para el conjunto de los indicadores evaluados. Suele manifestarse por un amplio repertorio de conductas [frentel a lo que se está evaluando [sobresalir] ... por [una] riqueza pedagógica [frente] ... al cumplimiento de los indicadores. (Mineduc, 2004a, p. 4)

En relación con los métodos de enseñanza y siguiendo las propuestas de Oller (2011), los de las ciencias sociales para el nivel de educación primaria deben responder al método científico, en particular con el método inductivo. Para la autora el método inductivo "permite que el alumnado formule explicaciones reconstruyendo su entorno social" (Oller, 2011, p. 167). Lo que se orienta a la construcción de un modelo que permite interpretar la realidad. Según Oller (2011), lo anterior se logra a partir del contraste o comparación de los fenómenos en estudio.

A partir de la interacción entre docente y estudiante asociada a la revisión que se realiza de los métodos de enseñanza, emergen las categorías que permitirán el análisis de las evidencias recopiladas. Siguiendo a Quinquer (2004), en el proceso de enseñanza aprendizaje de las ciencias sociales se reconocen métodos expositivos, interactivos y de aprendizaje individual. Para la autora, en los métodos expositivos el personal docente es el eje de la actividad en el aula escolar, dándose la participación del alumnado en diversos grados. Si bien el personal docente expone los contenidos, el estudiantado también participa en variados niveles dependiendo de la actividad implementada. Estos métodos, cercanos a los de la enseñanza tradicional, superan el aprendizaje memorístico cuando "el estudiante llega a dotarlos de significado porque relaciona los nuevos contenidos con lo que ya sabe y los integra en las estructuras del conocimiento que ya posee" (Quinquer, 2004, p. 104). La utilización de los métodos expositivos se evidencia, por ejemplo, en el uso de preguntas y respuestas.

En la clasificación de método interactivo, la autora plantea que niños y niñas "reelaboran los conocimientos por medio de la interacción con otros compañeros y con el docente" (Quinquer, 2004, p. 106). El método interactivo se evidencia a través de proyectos de aula y simulaciones, entre otras estrategias. Las simulaciones implican la reproducción de situaciones, que proporcionan al estudiantado un marco referencial, en función de los objetivos a alcanzar, y favorece la adquisición de nuevos conocimientos. En relación con el trabajo por proyectos, supone la determinación de un objetivo asociado a una temática de trabajo, planifica y ejecuta en equipo los pasos que permitan alcanzarlo.

Según Joyce y Weil (2000, p. 36), "un modelo de enseñanza no es sino una descripción de un ambiente de aprendizaje. Las descripciones tienen múltiples usos que van desde la planificación de currículos, cursos, unidades didácticas y lecciones hasta el diseño de material 
doi: http://dx.doi.org/10.15359/ree.23-3.18

URL: http://www.una.ac.cr/educare

CORREO: educare@una.cr

de enseñanza". Para los efectos de esta investigación, se revisan los modelos en cuanto su aplicación en el aula, esto es, respecto a la opción metodológica realizada por el profesorado. Así, de la propuesta de Joyce y Weil (2000) es posible reconocer como métodos de enseñanza de las ciencias sociales, la cooperación entre pares, está orientada a favorecer el trabajo con eficacia y "comprenden desde sistemas para enseñar a los alumnos a realizar tareas simples de aprendizaje en pareja, hasta modelos complejos destinados a organizar clases" (p. 39). Entre sus principales características, mencionan "la combinación del apoyo social [con un] aumento [de] la complejidad cognitiva producto de la interacción social" (Joyce y Weil 2000, p. 63), además de favorecer actitudes de compromiso y responsabilidad.

En la clasificación precedente se incluyen los juegos de roles y la investigación grupal. Para Joyce y Weil (2000), los juegos de roles permiten al alumnado el desarrollo de la empatía y de las habilidades sociales, favorecen la comprensión de las interacciones sociales y la resolución de problemas; de allí que se pueda considerar como una opción en los procesos de enseñanza de las ciencias sociales. Los autores caracterizan este método como "una situación de aprendizaje centrada en la experiencia y donde 'el aquí y el ahora' pasan a ser el contenido de enseñanza" (Joyce y Weil, 2000, p. 91). En cuanto a la investigación grupal, se define como un método "diseñado para llevar a los alumnos a definir problemas, explorar distintas perspectivas para abordarlos y estudiar juntos a fin de dominar la información, las ideas y las habilidades, desarrollando al mismo tiempo su competencia en el campo social" (Joyce y Weil, 2000, p. 39). Al personal docente le corresponde la organización y acompañamiento del proceso, convertido en facilitador del aprendizaje, transfiere el protagonismo al estudiantado.

En cuanto a los conceptos sociales claves, como se plantea en Castañeda (2016, p. 42, citando a Benejam, 1997, 1999), para que un concepto social pueda ser considerado como tal ha de cumplir con criterios precisos, entre estos:

Admitir un proceso de complejidad creciente que reconstruya la comprensión científica de la sociedad. Fundamentar la temática que la comunidad científica considera básica para la comprensión racional de la sociedad. Ser relevante para diversas disciplinas sociales. Permitir la estructuración y generación de conocimientos. Ser parte del currículum escolar. Tribbó (1999) agrega ser coherentes con los principios filosóficos y educativos en los que se basa y considerar una visión del mundo, de las ciencias referentes y su epistemología.

Para esta investigación, como se plantea en Castañeda (2016), se reconocen cinco conceptos sociales claves, entendiendo que estos poseen capacidad inclusiva y que no se limitan a la mera definición al permitir la articulación de diversos contenidos y conceptos que subyacen. Los conceptos sociales claves propuestos y sus subordinados se han determinado considerando tres colectivos. En primer término un colectivo formado por personal experto disciplinar del área 
de historia y ciencias sociales, que permitió la generación de una primera propuesta, conocida y validada por didactas del área de las ciencias sociales, de donde emerge una segunda propuesta que fue finalmente validada por profesorado destacado del sistema escolar.

Los conceptos sociales claves que emergen de esta propuesta y que serán considerados para los análisis posteriores son: tiempo, espacio, convivencia, identidad y cambio.

Tiempo, alude principalmente a la diversidad de manifestaciones para la comprensión del transcurso del tiempo asociado al devenir de la historia. Para este concepto clave se reconocen como subordinados aquellos que contribuyen a su comprensión como tiempo histórico, hecho histórico o proceso histórico. Es considerado eje de los procesos de enseñanza del pensamiento social (Cruz, 2004; Pagès, 2004b).

Espacio, este concepto refleja la relación de interdependencia permanente existente entre el sujeto con su entorno. Como conceptos asociados, aparecen territorio, barrio, comunidad y país. Al igual que el tiempo, la comprensión del espacio se reconoce como eje de los procesos de enseñanza del pensamiento social (Comes, 2004). Convivencia, se asocia a la formación para la vida en sociedad, entendida como espacio de concreción democrática. Los conceptos asociados a la convivencia se expresan como valores o actitudes que permiten y orientan la actuación de la persona en interrelación con otras, estos son: respeto, responsabilidad, solidaridad, libertad y reciprocidad (Benejam, 1999; Pagès, 2004a).

Identidad, el concepto clave identidad aparece equidistante de conceptos asociados como pertenencia y globalización, se busca conocer quiénes somos en un mundo global. Es reconocida como una necesidad que debiese ser considerada en la formación de las nuevas generaciones. La construcción de esa identidad puede plantearse de manera progresiva, así surgen conceptos como persona, familia, comunidad, inclusión y globalización (Benejam, 1999).

Cambio, como concepto social clave es reflejo de la complejidad de los fenómenos sociales y del desarrollo de la sociedad, se reconoce como una constante en la vida del ser humano y por extensión a la sociedad. Se evidencia en conceptos asociados como crisis, conflicto y revolución, los que son a su vez producto y origen del cambio (Benejam, 1999; Pagès, 2004a).

\section{Metodología}

La investigación se realiza desde un paradigma interpretativo, asociado a un diseño cualitativo, lo que implica, en palabras de Hernández et al. (2014, p. 9), basarse en "una lógica y proceso inductivo (explorar y describir, y luego generar perspectivas teóricas)". Para Latorre, del Rincón y Arnal (2005) el paradigma interpretativo "enfatiza la comprensión e interpretación de la realidad educativa desde los significados de las personas implicadas en los contextos educativos" (p. 42). 
doi: http://dx.doi.org/10.15359/ree.23-3.18

URL: http://www.una.ac.cr/educare

CORREO: educare@una.cr

El objetivo de la investigación es analizar los métodos de enseñanza de profesorado destacado del sistema escolar desde la perspectiva de los conceptos sociales claves. Como técnica de recogida de datos se utilizan focus group y observación no participante. El focus group se define como una conversación "diseñada para obtener información de un área definida de interés, en un ambiente permisivo, no directivo... los miembros del grupo se influyen mutuamente, puesto que responden a las ideas y comentarios que surgen en la discusión" Krueger (1991, p. 24). En este caso el focus group permitió el acercamiento al colectivo en estudio.

En relación con la observación no participante, aparece como principal característica que la realidad observada no se manipula ni se modifica (Ruiz, 2009, p. 125), tiene como requisitos en su recogida un objetivo claro y un trabajo planificado. La observación no participante permitió conocer el desarrollo de una secuencia didáctica para la asignatura de historia, geografía y ciencias sociales. En cada caso la observación consideró entre 8 y 10 horas pedagógicas por docente, lo que equivale a aproximadamente 450 minutos en cada caso.

El colectivo participante está constituido por cuatro docentes del nivel de educación básica, quienes se reconocen en el proceso de evaluación docente como profesoras destacadas. Son docentes de aula del sistema municipal de educación, realizan clases de historia, geografía y ciencias sociales en el nivel de educación primaria, poseen entre cinco y diez años de experiencia en aula y han accedido a participar voluntariamente de la investigación.

En cuanto a los criterios de rigor de la investigación que se presenta, entendido como "el grado de certeza de sus resultados, es decir, del conocimiento que ha producido" (Dorio, Sabariego y Massot, 2009, p. 287), responde al criterio de credibilidad que da cuenta de la validez interna del proceso: se realiza observación persistente del profesorado que se investiga, asistiendo a sus clases durante la implementación de una unidad didáctica, lo que supone diez clases en cada caso. En cuanto a su validez externa se cuenta con una descripción exhaustiva de las clases observadas, lo que permite el análisis de la información, a partir de la organización dada por los conceptos sociales claves y los métodos de enseñanza. En cuanto a la confirmabilidad de la información recogida, se utilizó el juicio experto tanto para validar la pauta del instrumento utilizado (focus group) como para la estructura seguida para la observación de clases. Se realiza así un ejercicio de reflexión permanente desde datos teóricos y empíricos.

El plan de análisis implementado considera en primer término el análisis de las evidencias del focus group, desde los aportes realizados y, principalmente, la disponibilidad personal e institucional para acceder a observar las clases implementadas se seleccionan cuatro personas reconocidas como personal docente destacado. La observación de clases se realiza desde el reconocimiento de la presencia de conceptos sociales en sus prácticas, así como de los métodos utilizados para abordarlos. Desde los referentes anteriores, se determina la presencia de conceptos sociales claves en los contenidos trabajados en el aula, así como los métodos que utiliza preferentemente el personal docente, lo cual permitió alcanzar el objetivo de análisis de sus métodos de enseñanza (Ver Figura 1). 


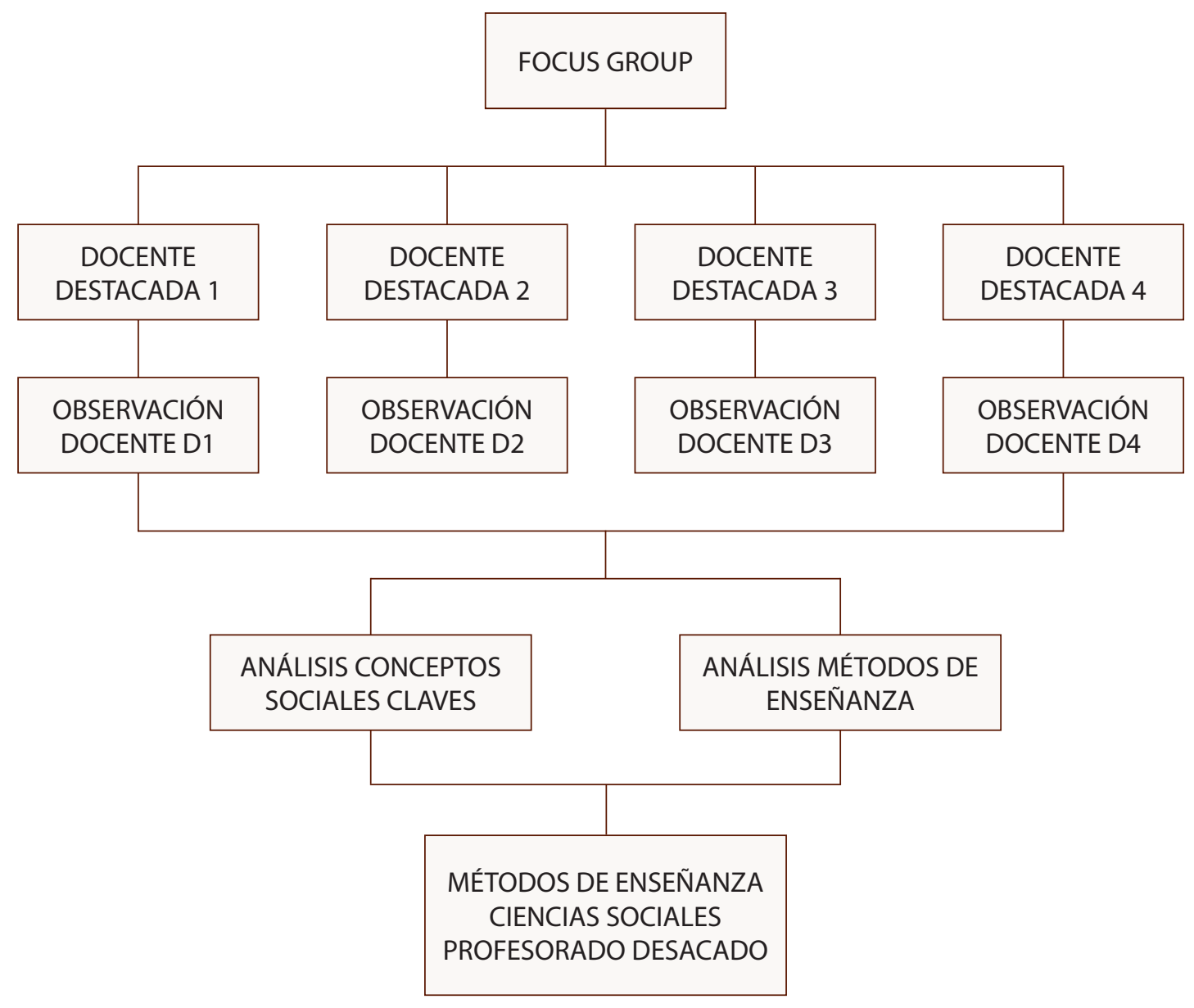

Figura 1: Plan de Análisis de la Información.

Nota: Elaboración propia.

\section{Resultados, análisis y discusión ${ }^{4}$}

Desde los resultados que emergen desde el focus group, es posible afirmar que el profesorado que participa de la investigación prioriza en sus procesos de planificación la selección de las metodologías. "Lo importante son la actividades, la selección de actividades lúdicas que los entusiasme y uno tiene que buscar, la parte histriónica, es muy importante" (DD1). "Yo planifico lo que me interesa que el alumno pueda comprender". (DD3). Complementariamente

${ }^{4}$ En el análisis de los fragmentos se utilizan las siguientes abreviaturas DD1 equivale a persona docente destacada 1; DD2 a persona docente destacada 2 y así sucesivamente. 
doi: http://dx.doi.org/10.15359/ree.23-3.18

URL: http://www.una.ac.cr/educare

CORREO: educare@una.cr

se observa frente a los conceptos sociales el proceso de complejidad creciente que es propio de los conceptos sociales, así como su pertenencia al curriculum escolar. (Benejam, 1997, 1999) "es importante el respeto, la participación, pero como lo hacemos práctico si en el fondo nos vemos haciendo clases enfocados en el concepto y no en la acción" (DD3)

El análisis se presenta desde cada uno de los conceptos sociales claves propuestos para el estudio para dar cuenta de su presencia explícita o implícita en las clases de ciencias sociales observadas, se ejemplifica con fragmentos relevantes seleccionados desde las instrucciones 0 comentarios realizados por el profesorado en el desarrollo de las unidades didácticas.

Concepto social clave tiempo. Se puede reconocer su presencia explicita e implícita en las evidencias analizadas. La presencia es vidente cuando responde al contenido escolar. Así, para el objetivo aplicar conceptos relacionados con el tiempo, se encuentra la siguiente evidencia: "qué día está antes del martes? Piensen primero. Estudiantes responden: lunes. ¿Qué día está después del viernes? Estudiantes: sábado. Bien, muy bien" (DD2). La referencia explícita responde principalmente a un contenido instrumental que busca la aplicación de parte del estudiantado. La referencia implícita se produce al encontrar referentes temporales aislados como es el caso de: "Vamos a irnos 1810 parte la Independencia, comienza y comienza la Patria Vieja con la Primera Junta Nacional de Gobierno, vamos a ir viendo" (DD1). Así, entonces, los fragmentos evidencian que el abordaje del tiempo queda solo en un carácter nominal, no encontrándose en las clases observadas referencias que apelen a su comprensión a través de sus conceptos subordinados como hecho o proceso.

Concepto social clave espacio. El análisis de la evidencias a lo largo de las sesiones observadas permite encontrar escasas referencias de la consideración del espacio, asociado exclusivamente al trabajo de mapas al estilo de "Primero Cristóbal Colón con rojo, segundo Hernando de Magallanes que da la vuelta al mundo con Sebastián Elcano que lo termina y luego, con verde Américo Vespucio que llega a América, al sur de América (DD4). Si se considera que tiempo y espacio son reconocidos como claves para la comprensión del mundo y de los procesos históricos y sociales, es relevante su escasa presencia como contexto de los procesos estudiados en el aula escolar, donde el espacio aparece como limitado a un trabajo de mapa que no necesariamente permite reconocer sus características y su efectiva incidencia en la comprensión del mundo. De ello da cuenta, además, el siguiente fragmento "Establecieron relaciones diplomáticas con la junta de Buenos Aires? ¿Dónde está Buenos Aires?" (DD1)

Por otro lado, cuando se hace referencia explícita a conceptos subordinados como barrio, este aparece más como elemento de contexto que permite interpretaciones posteriores. Así se encuentran fragmentos como "¿Qué relación tiene mi barrio con esta imagen? ¿Qué relación tienen esas dos cosas? ¿Por qué dice mi barrio y por qué sale esta imagen?" (DD3). Como se aprecia, al igual que en el caso anterior, no existe un trabajo asociado a la comprensión del concepto, sino más bien a su consideración como referente. 
Concepto social clave convivencia. En este caso se encuentran evidencias explícitas asociadas tanto a la comunidad como a los poderes del Estado. Así se tiene "¿Qué hace el Congreso Nacional? Estudiante: resumir las leyes y autorizar al reino...Docente ¿Qué son las leyes? Estudiante: son reglas. Docente: Son reglas de comportamiento cierto, se crea un reglamento. A ver y acá me empieza a parecer extraño a mí, si nosotros pretendemos devolverle nuestra colonia a España cuando vuelva el rey para qué estamos creando un reglamento interno" (DD1). "Él es una familia, ¿puede ser una familia solito? ¿Podría ser una familia solito? Estudiante: no. Docente: No, por qué no, haber. Estudiante: no puede estar solito porque si no, no tendría acompañante. Ya, la alumna me dice que no puede estar solito porque si no, no tendría acompañante. ¿Existirán familias de uno? (DD2).

En ambos casos se aprecia que desde los diálogos planteados se aborda la formación para la vida en sociedad, entendida como espacio de concreción democrática. En el primer caso no solo se prioriza el conocimiento, sino que el personal docente plantea una interrogante que busca generar diálogo en el estudiantado, si bien el proceso de cuestionamiento emerge desde el docente o la docente, hay una clara intencionalidad orientada a la comprensión. Lo mismo ocurre en el segundo caso, en donde la docente claramente contribuye a ampliar una visión de composición tradicional de la familia, llevando a sus estudiantes a preguntarse si una persona sola constituye familia. Esta reflexión que inicia la docente se asocia al concepto de convivencia propuesto, reconociendo al sujeto en diálogo con la sociedad.

Concepto social clave cambio. Asociado a la complejidad de los fenómenos sociales, se encuentran evidencias de su tratamiento implícito: "Después de esos años de tinieblas que Chile pertenece a España, vino la luz, viene esta luz de la llustración, que nos viene, cierto, a dar estas ideas de libertad. Mira qué bonito lo que hacen los hermanos Carrera. La primera bandera nacional, el primer escudo nacional" (DD1). El concepto social se expresa a través de la comparación entre dos momentos históricos, así el cambio se aborda visualizando elementos concretos, "bandera" y metafóricos "años de tinieblas". El cambio como concepto social clave aparece asociado, principalmente, a hacer consciente al alumnado de la existencia de momentos históricos que se diferencian desde la particularidad. De lo anterior también da cuenta el siguiente fragmento "el indígena vivía en su territorio, tranquilito, con su familia, su alimentación, estaban en paz y de repente llega el hombre europeo con la tecnología, con otra forma de pensar y ver el mundo. Por eso hay una duda si ellos realmente son descubridores o es una interrupción del mundo indígena, o un encuentro, ahí tiene varios nombres, después lo vamos a discutir" (DD4). En el fragmento precedente, al igual que en el anterior, el cambio aparece asociado a la comparación, más que a la constatación del hecho que lo origina.

Concepto social clave identidad: En la búsqueda de saber quiénes somos en un mundo global, la identidad aparece abordada implícitamente en la clase de historia cuando se hace referencia a etapas de la historia nacional "fuimos de más a menos, primero descubrimiento de 
doi: http://dx.doi.org/10.15359/ree.23-3.18

URL: http://www.una.ac.cr/educare

CORREO: educare@una.cr

América, luego conquista de América y ahora descubrimiento y conquista de Chile, de más a menos, a lo puntual, a nuestro territorio" (DD4). Si bien no hay una referencia explícita a la identidad, sí aparecen referentes que leídos en contexto permiten acercar al estudiantado a la pertenencia a un territorio asociada al reconocimiento de elementos comunes.

En cuanto al análisis desde los métodos para la enseñanza utilizados por el grupo docente, los conceptos sociales claves se evidencian de forma explícita o implícita, con un claro predominio del método expositivo como lo plantea Quinquer (2004): existe un intento por superar el aprendizaje memorístico que se orienta a que el estudiantado, frente a los nuevos contenidos, llegue a "dotarlos de significado porque relaciona los nuevos contenidos con lo que ya sabe y los integra en las estructuras del conocimiento que ya posee" (Quinquer, 2004, p. 103). En esa búsqueda de dotar de significado encuentran evidencias tales como "¿En qué año estamos? ... ¿Qué día? ... ¿Qué mes?... Esto que hacemos todos los días lo voy a empezar a hacer así, desordenado... al final se lo van a aprender de memoria, quiero que comprendan lo que está ahi" (DD2). En el fragmento, a pesar de que hay un claro predominio docente, se trata de intencionar la búsqueda de significado por sobre la respuesta automática por parte del estudiantado.

A partir de la observación realizada es posible afirmar que se encuentran evidencias de trabajo en el aula que pueden ser asociadas a simulaciones. Para Quinquer (2004), las simulaciones implican la reproducción de situaciones, en las que se proporciona al estudiantado un marco referencial, en función de los objetivos a alcanzar, para favorecer la adquisición de nuevos conocimientos. Un ejemplo de ello es la simulación a partir de una interrogante "¿Qué harían si una compañera se dedica a molestar en clases, bota basura e interrumpe en reiteradas ocasiones?" (DD3). En este caso, la docente plantea una pregunta hipotética, simulación, frente a la cual el estudiantado discute posibles alternativas para darle respuesta. Lo mismo ocurre frente a los contenidos de la historia de América: "¿Qué creen que habrá pensado estas dos personas que nunca antes se habían visto?, ¿Cuál habrá sido, como habrá sido el encuentro de este español que nunca había visitado este continente y este indígena que nunca había visto a este español?" (DD4). En ambos fragmentos se trata, a través de la situación simulada en el relato docente, buscar un posicionamiento estudiantil frente a lo planteado.

Si bien, el objetivo de estas líneas es solo evidenciar la presencia de este método en el aula en el abordaje de los conceptos sociales claves, es posible señalar que, en la enseñanza de las ciencias sociales, la utilización de estas situaciones simuladas permite, en palabras de Quinquer (2004), profundizar en un marco referencial para la adquisición de conocimientos. Así, los ejemplos proporcionados se orientan a una comprensión de la realidad desde más de una perspectiva -quien interrumpe y a quien se interrumpe, los grupos aborígenes y los grupos españoles- que contribuye, de forma incipiente, a comprender la complejidad de los fenómenos sociales. 
En el caso del método de enseñanza asociado a la investigación grupal, que se orienta, según plantean Joyce y Weil (2000), al dominio de la información y al desarrollo de competencias sociales, como muestra el siguiente fragmento:"como estábamos viendo en la noticia anterior ¿qué es lo que era? ¿cuál era su rol? hijos, acá son estudiantes... lo que yo les voy a preguntar es ¿cuál de esas dos situaciones?, ¿Porquéla que tienen ahísería la ideal? (DD3). El planteamiento de las interrogantes propuestas por el docente, para favorecer un espacio de discusión grupal, permite abordar en este caso el concepto de identidad a la base de los contenidos tratados, así como desde el intercambio entre el estudiantado: efectivamente, se desarrollan las competencias sociales.

El siguiente ejemplo en el cual la docente organiza el trabajo en equipo de sus estudiantes complementa el anterior "a cada grupo le va a tocar la conquista del imperio azteca o inca ¿qué debe hacer? recortar las imágenes, y luego armarán una historia en línea de tiempo, ... cuentan la historia con palabras propias...Alguien se va a dedicar a recortar, pintar, la idea es que se distribuyan las tareas... las imágenes están desordenadas, ustedes deberán darle el sentido" (DD4). En este caso, con mayor claridad se incentiva la distribución de tareas entre cada integrante y se plantea la situación a investigar o resolver, pudiendo reconocerse, en la base, el concepto social de identidad, asociado a los orígenes de las sociedades americanas.

\section{Conclusiones}

La investigación de la cual da cuenta este artículo se centra en analizar los métodos de enseñanza utilizados por profesorado destacado del nivel de educación primaria desde la perspectiva de los conceptos sociales claves. Como primera idea, es necesario señalar que esta investigación ratifica que los conceptos sociales claves subyacen a los contenidos presentes en los programas de estudio escolares y que los propuestos: tiempo, espacio, convivencia, cambio e identidad pueden ser reconocidos a la base de los contenidos abordados en el aula escolar. Que los conceptos sociales claves puedan ser reconocidos en los contenidos escolares no implica que sean abordados explícitamente. Sin embargo, en la búsqueda de una comprensión que supere la definición o el dato, se hace necesario visibilizarlos. Esto adquiere particular importancia en lo que se refiere a tiempo y espacio, considerados elementos claves en la enseñanza de las ciencias sociales, en particular de la historia que predomina en la enseñanza escolar, dado que todo hecho o proceso histórico se implica un diálogo permanente entre ambos conceptos. En relación con los conceptos subordinados a los conceptos sociales, estos aparecen escasamente referenciados sin que, necesariamente, se profundice en estos mismos, como en el caso de barrio como concepto subordinado a espacio.

Los métodos de enseñanza implementados en las clases de ciencias sociales por el profesorado destacado que participa del estudio evidencian una perspectiva tradicional, asociada principalmente a un método expositivo. Sin embargo, esa opción metodológica no descarta al estudiantado, donde, desde un rol participativo, cede el protagonismo a su docente en su rol de guía del aprendizaje. 
doi: http://dx.doi.org/10.15359/ree.23-3.18

URL: http://www.una.ac.cr/educare

CORREO: educare@una.cr

La perspectiva, principalmente expositiva-participativa, evidenciada por personal docente destacado del nivel de educación primaria del sistema escolar, permite concluir la necesidad de una evolución hacia una mayor y mejor participación de niños y niñas, donde en la clase de ciencias sociales se supere el discurso como la principal forma de lograr y constatar aprendizajes.

\section{Referencias}

Benejam, P. (1997). La selección y secuenciación de los contenidos sociales. En P. Benejam y J. Pagès (Coords.), Enseñar y aprender ciencias sociales, geografía e historia en la educación secundaria (pp. 71-96). Barcelona: Horsori.

Benejam, P. (1999). La oportunidad de identificar conceptos clave que guíen la propuesta curricular de las ciencias sociales. Íber: Didáctica de las ciencias sociales, geografía e historia, 21, 5-12.

Carretero, M., Castorina, J. A., Sarti, M., Van Alphen, F. y Barreiro, A. (2013). La construcción del conocimiento histórico. Revista Propuesta Educativa, 1(39), 13-23. Recuperado de http:// www.redalyc.org/articulo.oa?id $=403041710003$

Castañeda, M. (2016). Conceptos sociales claves para la organización curricular de la enseñanza de historia en educación básica. En V. Acuña (Comp.), XXIII Encuentro nacional de investigadores en educación. Transformaciones de la educación: Una mirada desde la investigación (pp. 4146). Valparaíso: UPLACED. Recuperado de https://issuu.com/humbertozapataolivares/ docs/2016 1123 eninupla2016 posteres/291

Comes, P. (2004). La enseñanza de la geografía y la construcción del concepto de espacio. En P. Benejam y J. Pagès (Coords.), Enseñar y aprender ciencias sociales, geografía e historia en la educación secundaria (pp. 169-187). Barcelona: Horsori.

Cruz, M. A. (2004). Tiempo histórico y su tratamiento didáctico. En M. Domínguez (Coord.), Didáctica de las ciencias sociales para primaria (pp. 233-260). Madrid: Pearson Educación.

Dorio, l., Sabariego, M. y Massot, I. (2009). Características generales de la metodología cualitativa. En R. Bisquerra (Coord.) Metodología de la investigación educativa. (pp. 275-292). Madrid: La Muralla.

Hernández, R., Fernández, C. y Baptista, P. (2014). Metodología de la investigación. México: McGraw- Hill. Recuperado de http://www.esup.edu.pe/descargas/dep investigacion/ Metodologia\%20de\%20la\%20investigaci\%C3\%B3n\%205ta\%20Edici\%C3\%B3n.pdf

Joyce, B. y Weil, M. (2000). Modelos de enseñanza. Barcelona: Gedisa.

Krueger, R. A. (1991). El grupo de discusión: Guía práctica para la investigacióna. Madrid: Pirámide. 
Latorre, A., del Rincón D. y Arnal, J. A. (2005). Bases metodológicas de la investigación educativa. Barcelona: Ediciones Experiencia.

Martorella, P. (1993). Enseñanza de conceptos. En J. M. Cooper (Coord.), Estrategias de enseñanza. Guía para una mejor instrucción (pp. 229-278). México: Limusa.

Ministerio de Educación (Mineduc). (2003). Marco de la Buena Enseñanza. Santiago: Centro de Perfeccionamiento e Investigaciones Pedagógicas. Recuperado de https://www. ayudamineduc.cl/ficha/marco-para-la-buena-ensenanza-5

Ministerio de Educación (Mineduc). (11 de junio de 2004a). Decreto 192. Aprueba reglamento sobre evaluación docente. Recuperado de https://www.leychile.cl/N?i=239053\&f=2005-06-11\&p=

Ministerio de Educación (Mineduc). (9 de abril de 2004b). Resolución 11025 Exenta. Aprueba Marco para la Buena Enseñanza. Recuperado de https://www.leychile.cl/N?i=229900\&f=2004-09-04\&p=

Ministerio de Educación (Mineduc). (2012). Programa Historia, geografía y ciencias sociales. Santiago: Autor.

Oller, M. (2011). Métodos y estrategias para la enseñanza y aprendizaje de las ciencias sociales. En A. Santisteban y J. Pagès (Coords.), Didáctica del conocimiento del medio social y cultural en la educación primaria (pp. 163-183). Madrid: Síntesis.

Pagès, J. (2004a) La formación del pensamiento social. En P. Benejam y J. Pagès (Coords.), Enseñar y aprender ciencias sociales, geografía e historia en la educación secundaria (pp. 151-168). Barcelona: Horsori.

Pagès, J. (2004b) El tiempo histórico. En P. Benejam y J. Pagès (Coords.), Enseñary aprender ciencias sociales, geografía e historia en la educación secundaria (pp. 189-207). Barcelona: Horsori.

Pozo, J. I. (1992). El aprendizaje y la enseñanza de hechos y conceptos. En P. Gil (Ed.), Los contenidos de la reforma (pp. 19-79). Madrid: Santillana.

Quinquer, D. (2004). Estrategias de enseñanza: Los métodos interactivos. En P. Benejam y J. Pagès (Coords.), Enseñar y aprender ciencias sociales, geografía e historia en la educación secundaria (pp. 97-122). Barcelona: Horsori.

Ruiz, J. I. (2009). Metodología de investigación cualitativa. Bilbao: Universidad de Deusto.

Santisteban, A. (2010). La formación de competencias de pensamiento histórico. Revista Clio \& asociados, 14, 34-56. doi: https://doi.org/10.14409/cya.v1i14.1674

Taba, H. (1971). Elaboración del curriculum. Buenos Aires: Troquel.

Tribó, G. (1999). Los conceptos clave en las propuestas curriculares. Íber: Didáctica de las ciencias sociales, geografía e historia, 21, 73-88. 